\title{
INTERACCIONES ESPACIALES Y TOPOLOGÍA EMPRESARIAL: LA CIRCULACIÓN DE MATERIAS PRIMAS E INSUMOS INDUSTRIALES EN TANDIL, BUENOS AIRES, ARGENTINA
}

Josefina Di Nucci ${ }^{1}$

\section{Resumen}

La industria, una de sus ramas o una empresa en particular produce una lógica territorial cuya manifestación visible es una topología; es decir, puntos y áreas que las empresas seleccionan y que conforman su base material de existencia para la producción, circulación y consumo. Estos son los fijos necesarios para realizar su actividad, es decir, su división del trabajo. Estudiamos ese aspecto visible de las empresas a partir de un análisis de las interacciones espaciales de Tandil con diferentes núcleos urbanos como resultado de la circulación de flujos materiales para la producción, en particular la compra de las materias primas y los insumos industriales. Esto nos permite ver la división territorial del trabajo de la actividad industrial de Tandil y de sus firmas, considerando que cada división territorial del trabajo se asocia a un momento del proceso de urbanización y del período de la historia que estamos viviendo.

Palabras claves: Interacción espacial; topologías de las empresas; materias primas e insumos; Tandil.

\section{INTERAÇÃO ESPACIAL E TOPOLOGIA DAS EMPRESAS: CIRCULAÇÃO DAS MATÉRIASS-PRIMAS E INSUMOS INDUSTRIAIS EM TANDIL (BUENOS AIRES)}

\section{Resumo}

A indústria, em seus ramos ou a partir de uma empresa particular produz uma lógica territorial cuja manifestação visível é uma topologia; ou seja, pontos e áreas que as empresas se apropriam e constituem a sua base material da existência para a produção, circulação e consumo. Estes são o fixo necessário para realizar a sua atividade, ou seja, sua divisão do trabalho. Nós estudamos o aspecto visível de empresas a partir de uma análise das interações espaciais de Tandil com centros urbanos diferentes, como resultado da circulação de fluxos de materiais para a produção, incluindo a compra de matérias-primas e insumos industriais. Isso nos permite ver a divisão territorial do trabalho na atividade industrial em Tandil e suas firmas, considerando que cada divisão territorial do trabalho está associado com um momento de processo de urbanização e com o período da história que estamos vivendo.

Palavras-chaves: Interação espacial; topologías das empresas; matérias-primas e insumos; Tandil.

\footnotetext{
${ }^{1}$ Doutora em Geografia pela Universidad Nacional Del Sur. Professora e Diretora do Departamento de Geografía da Facultad de Ciencias Humanas (FCH) da Universidad Nacional Del Centro de La Prov. de Buenos Aires (UNICEN). Email: josedinucci@yahoo.com
} 


\title{
SPATIAL INTERACTION AND ENTERPRISES TOPOLOGY: CIRCULATION OF RAW MATERIALS AND INDUSTRIAL SUPPLIES IN TANDIL (BUENOS AIRES)
}

\begin{abstract}
:
The industry, one of its branches or a particular company produces a territorial logic whose visible manifestation is a topology; scilicet, points and areas that firms select and make their material basis of existence for the production, circulation and consumption. These are the fixed necessary to perform its activity, that is, its division of work. We study the visible aspect of the firms from an analysis of the spatial interactions of Tandil with different urban centers as a result of the circulation of material flows for production, including the purchase of raw materials and industrial inputs. This allows us to see the territorial division of work in industrial activity in Tandil and their firms, considering that each territorial division of work is associated with a moment of urbanization process and the period of history we are living.
\end{abstract}

Keywords: Spatial interaction; topologies enterprises; raw materials and inputs; Tandil.

\section{INTRODUCCIÓN}

Si consideramos el proceso general de producción y reproducción del capital en todas y cada una de sus fases, producción, distribución, intercambio y consumo, vemos como estos momentos se llevan a cabo de manera desagregada pero articulada en el espacio (ARROYO y DA CRUZ, 2015). Es esta la divisibilidad del espacio que es explicada por diferentes nociones o concepciones como la de división territorial del trabajo. Hay en nuestros países y en nuestras ciudades un enrejado de divisiones del trabajo, una superposición de topologías corporativas, lo cual lleva a afirmar que división territorial del trabajo es un concepto plural (SILVEIRA, 2008).

La industria como actividad económica, una de sus ramas específicas o una empresa en particular produce una lógica territorial cuya manifestación visible es una topología; es decir, los puntos y áreas que las empresas seleccionan y que conforman su base material de existencia para la producción, circulación y consumo. Son estos los fijos necesarios para realizar su actividad. "Esta es su propia división del trabajo: una verdadera topología..." (SILVEIRA, 2007, p. 15). Como indica esta autora "los movimientos entre estos fijos son los flujos, es decir, el circuito espacial de producción..." (SILVEIRA, 2007, p. 20). Arroyo y Da Cruz (2015, p. 9) remarcan que la divisibilidad del espacio no es absoluta "dado que las instancias productivas están articuladas a través de la circulación".

Entonces existe un lado o aspecto visible de la división territorial del trabajo junto a uno invisible bastante más complejo y definido como: 
[...] el conjunto de operaciones que envuelve lugares, el sistema de acciones propio de la empresa, que le da una posición ventajosa a escala global y le posibilita, gracias a la técnica contemporánea, una comunicación en tiempo real y el uso de instrumentos financieros a tal punto perfeccionados que dinero e información se confunden (SILVEIRA, 2007, p. 20).

Vamos a estudiar ese aspecto visible de las empresas, o de la industria tandilense en particular, a partir de la circulación asociada al intercambio de mercaderías, al movimiento generado por los flujos de materias primas e insumos entre las industrias de Tandil y los nodos urbanos con los cuales esta ciudad se conecta relacionado esto, en muchos casos, con la especialización productiva de los lugares. Entonces, la división territorial del trabajo de las industrias genera articulaciones entre los núcleos urbanos que, muchas veces, concentran actividades diversas o específicas, especialmente observable a partir de la circulación e intercambios de objetos materiales, como mercancías, personas y dinero, aunque también inmateriales como información, por ejemplo. De esta manera, se van reforzando las diferenciaciones entre los lugares de acuerdo a las funciones o papeles urbanos que poseen en dicha red más o menos articulada. Santos (1996, p. 62) señala que "más circulación y más movimiento permiten la profundización de la división territorial del trabajo y esto crea, a su vez, más especialización del territorio".

Este autor afirma también que "el sistema de ciudades constituye el armazón del espacio" (Santos, 1996, p. 58) el esqueleto económico, político, institucional y socio-cultural de un país. "La red urbana es un conjunto de aglomeraciones que producen bienes y servicios junto con una red de infraestructura de soporte y los flujos que, a través de esos instrumentos de intercambio, circulan entre las aglomeraciones" (SANTOS, 1996, p. 57). En este sentido Corrêa (1989, p. 48) afirma que:

[...] la red urbana se constituye simultáneamente en un reflejo de y una condición para la división territorial del trabajo. Es un reflejo en la medida que, en razón de ventajas locacionales diferenciadas, se verifica una jerarquía urbana y una especialización funcional definitorias de una compleja tipología de centros urbanos.

La red de ciudades, actuando de manera articulada según sus funciones, se convierte en una condición para el desarrollo de la división territorial del trabajo. Es la red urbana la que vuelve viable y posible a la producción, la circulación y el consumo (CORRÊA, 2004).

Observando la jerarquía urbana, el papel de las grandes ciudades es central porque son por excelencia los centros de producción y de consumo y, por lo tanto, también los grandes 
centros de distribución y los grandes nudos de circulación. Concentran el comercio mayorista interno y el comercio de exportación e importación (SANTOS, 1996). "Pero es efectivamente debido a la acción de los centros de acumulación del capital, las grandes metrópolis cabeceras de redes urbanas de extensión mundial o nacional, que la división territorial del trabajo aparece condicionada por la red urbana" (CORRÊA, 1989, p. 50).

Los intercambios o circulación valorizan lugares y desvalorizan otros (ARROYO, 2015). Para que esta circulación se pueda efectivizar es necesaria la existencia de las ciudades, como puntos de territorio constituyentes de las redes urbanas. "Estos centros urbanos se apropian del valor excedente que circula y crea nuevos valores" (CORRÊA, 1989, p. 52). Así, las ciudades que forman parte de esta red urbana existente alrededor de la industria tandilense se apropian de manera diferencial del valor excedente.

Nos interesa aquí ver las interacciones espaciales (CORRÊA, 1997) entre Tandil, como lugar de producción, de elaboración misma de productos industriales y otros centros urbanos de la provincia de Buenos Aires, del resto de Argentina y del mundo, a partir de analizar los intercambios comerciales, los flujos de mercaderías derivados de dicha actividad. Para ello observaremos el tipo y procedencia de materias primas e insumos que compra la industria tandilense. En algún sentido esto nos permite ver la división territorial del trabajo de la actividad industrial de Tandil y de sus firmas, considerando que cada división territorial del trabajo se asocia a un momento del proceso de urbanización y del período de la historia que estamos viviendo.

Existe una historia de la red urbana argentina y de la provincia de Buenos Aires con jerarquías urbanas también heredadas. Esta red de ciudades nos serviría como base o plataforma para estudiar como se superpone a ella la división territorial de trabajo de la actividad industrial. En este caso miramos esta relación a partir de una ciudad media del centro de la provincia de Buenos Aires: Tandil.

En algún sentido nos referimos al impacto espacial de la actividad industrial, de su topología empresarial (forma en que se organiza) que se superpone a la red urbana históricamente constituida.

Para la realización de este trabajo contamos con los datos del Relevamiento Industrial de Tandil (CIG/ Municipio de Tandil, 2013) sobre un total de 670 empresas que representan el $96 \%$ del total de la existentes en esta ciudad. Se han seleccionado las siguientes variables: Materias primas utilizadas según orden de importancia y lugar de procedencia del proveedor 
de las mismas e, - Insumos necesarios para el proceso de producción y localidades de procedencia.

Resulta importante realizar algunas aclaraciones metodológicas respecto a cómo se tomaron los datos y cómo se utilizan en este trabajo. La pregunta sobre la adquisición de materias primas, fue respondida por el $88,5 \%$ del total de empresas encuestadas (596 empresas de un total de 670). Para este trabajo se tomaron todas las respuestas de cada una de las encuestas, lo cual lleva a que la cantidad de respuestas totales sobre cuál es la ciudad de donde provienen las materias primas es de 1630. Por otro lado, los encuestados respondieron sobre cuál es el porcentaje asignado a esa materia prima y localización, por lo cual también se tomó esta respuesta haciendo una suma de porcentajes por ciudad y que, en algún sentido, nos muestra el volumen del flujo de materias primas.

La pregunta respecto a adquisición y procedencia de insumos fue respondida por menor cantidad de empresas (520 de 670 totales) que representan el 77,6\% del total de las mismas. La cantidad de respuestas totales sobre cuál es la ciudad de donde provienen los principales insumos es de 1541 respuestas.

En este artículo se realiza un análisis de las interacciones espaciales de Tandil con diferentes núcleos urbanos a partir de la circulación de flujos materiales para la producción industrial, en particular la compra de las materias primas y los insumos industriales.

\section{LA CIRCULACIÓN DE MATERIAS PRIMAS E INSUMOS PARA LA PRODUCCIÓN INDUSTRIAL}

Observar la procedencia de la materia prima e insumos utilizados en la industria tandilense, o dicho de otro modo, el lugar donde la industria de Tandil compra sus materias primas e insumos de producción permite mostrarnos una primera aproximación del análisis a las interacciones espaciales interurbanas resultado de esta actividad económica. Según Corrêa (1997, p. 279) "las interacciones espaciales constituyen un amplio y complejo conjunto de desplazamientos de personas, mercaderías, capital e información sobre el espacio geográfico". Las mismas pueden tener diferentes intensidades, frecuencias, ocurrencias y, en especial, diferentes propósitos. La circulación de mercancías o productos entre diferentes ciudades, entre fábricas y tiendas, las exportaciones y las importaciones, son ejemplos típicos de interacciones espaciales. 
Para organizar la información hemos seleccionado un criterio de escala utilizando las siguientes unidades espaciales: Tandil; Región Metropolitana de Buenos Aires; resto de la provincia de Buenos Aires; resto de la Argentina y resto del mundo.

Se presentan en la tabla 01 los datos generales de las variables seleccionadas, los cuales se irán analizando y desdoblando a lo largo de este trabajo.

\section{Tabla 01: Procedencia de Materias primas e insumos para la industria de Tandil, por} grandes unidades espaciales (\%). 2013.

\begin{tabular}{l|cc}
$\begin{array}{l}\text { Unidades } \\
\text { espaciales }\end{array}$ & $\begin{array}{c}\text { Materias } \\
\text { primas }\end{array}$ & Insumo \\
\hline Tandil & 63,5 & 64,9 \\
RMBA & 17,7 & 20,2 \\
$\begin{array}{l}\text { Resto Provincia de } \\
\text { Buenos Aires }\end{array}$ & 11,7 & 9,9 \\
Resto Argentina & 5,2 & 2,6 \\
Resto del Mundo & 2,0 & 2,4 \\
\hline Total & $\mathbf{1 0 0}$ & $\mathbf{1 0 0}$
\end{tabular}

Fuente: elaboración personal en base al Relevamiento Industrial Tandil. 2013. CIG-FCH-UNCPBA y Municipio de Tandil.

En la tabla 01 observamos la procedencia de la materia prima e insumos poniéndose en evidencia la fuerte horizontalidad territorial de la industria de Tandil ya que el 63,5\% de las materias primas son obtenidas localmente ya sea porque se extraen o elaboran en este partido o porque son adquiridas en comercios mayoristas de la ciudad. Esto evidencia también la importancia de las actividades concatenadas a la industria en esta ciudad media. Un valor muy similar corresponde a la adquisición de insumos necesarios para la fabricación de productos industrializados con casi el $65 \%$ de los mismos comprados al interior del partido.

La especialización industrial de Tandil en las ramas alimenticia y metalmecánica y la importancia de las empresas de tamaños menores, micro o pequeñas (familiares especialmente), es otra posible explicación del fuerte componente local de las compras de materias primas e insumos de las industrias tandilenses. Así, según los datos presentados por 
Lan (2014) la rama (31) alimenticia y bebidas de Tandil compra el 80,1\% de las materias primas que utiliza en el Partido de Tandil y las ramas de la metalmecánica (37 y 38) lo hacen en un $77,8 \%$ y $64,6 \%$ respectivamente. Otras ramas industriales con un fuerte componente local en sus compras de materias primas son las 33 (Industria de la madera y productos de la madera, incluido muebles) y la 36 (Fabricación de productos minerales no metálicos) con un $58,8 \%$ y un $53,8 \%$ respectivamente.

Observamos en la tabla 1 la destacable concentración de compras de materias primas e insumos en la metrópolis de Buenos Aires, siendo un poco más elevado el porcentaje de insumos $(20,2 \%)$ que el de materia prima $(17,7 \%)$. Si bien se observa que prácticamente todas las ramas de actividad se relacionan con la RMBA en sus compras debemos destacar algunos tipos de producciones como las textiles, prendas de vestir e industrias del cuero (rama 32). Según la información con la que contamos y en relación a esta rama queremos destacar algunos productos adquiridos en Buenos Aires: telas, hilos, lonas y cueros, especialmente.

La fuerza de atracción de la metrópolis, como centros de producción, distribución, comercialización y consumo, se pone en invidencia al igual que la dependencia que las ciudades de menor jerarquía urbana poseen respecto a ella especialmente en la compra de todo tipo de productos tanto para consumo productivo como para consumo consumptivo.

\section{Las interacciones espaciales a escala provincial}

Tandil posee también una fuerte relación con su región; como puede observarse en la tabla 1 el 11,3\% de las materias primas necesarias y el 9,9\% de los insumos son adquiridos en la provincia de Buenos Aires (sin RMBA). Resulta interesante detenernos ahora en esta escala interurbana diferenciando provincia de Buenos Aires del resto de Argentina. Para poder observar la relación entre la división territorial del trabajo y la red urbana se ha desagregado la información por localidad, presentando en la tabla 02 y los mapas 01 y 02 , la procedencia de estos flujos según localidades de la provincia de Buenos Aires. 
Tabla 02: Procedencia de Materias primas e insumos de la Industria de Tandil, por localidades de la provincia de Buenos Aires (\%) 2013.

\begin{tabular}{|c|c|c|}
\hline Localidades & $\begin{array}{c}\text { Materias } \\
\text { primas }\end{array}$ & Insumo \\
\hline Mar del Plata & 6,32 & 5,67 \\
\hline Balcarce & 0,86 & 0,52 \\
\hline Olavarría & 0,67 & 1,04 \\
\hline Bahía Blanca & 0,61 & 0,39 \\
\hline Saladillo & 0,55 & 0,07 \\
\hline Necochea & 0,43 & 0,13 \\
\hline Benito Juárez & 0,18 & 0,26 \\
\hline San Nicolás & 0,18 & 0 \\
\hline Tres Arroyos & 0,18 & 0 \\
\hline Bragado & 0,12 & 0,07 \\
\hline Campana & 0,12 & 0 \\
\hline Cañuelas & 0,12 & 0 \\
\hline Villa Gesel & 0,12 & 0 \\
\hline 9 de Julio & 0,06 & 0 \\
\hline Azul & 0,06 & 0,13 \\
\hline Baradero & 0,06 & 0 \\
\hline Chivilcoy & 0,06 & 0 \\
\hline Colón & 0,06 & 0 \\
\hline Escobar & 0,06 & 0 \\
\hline Junín & 0,06 & 0,26 \\
\hline La Plata & 0,06 & 0,20 \\
\hline Gral. Madariaga & 0,06 & 0 \\
\hline Pergamino & 0,06 & 0,07 \\
\hline Pigué & 0,06 & 0,07 \\
\hline Pila & 0,06 & 0,07 \\
\hline Pilar & 0,06 & 0 \\
\hline Rauch & 0,06 & 0,20 \\
\hline Rojas & 0,06 & 0,07 \\
\hline Roque Pérez & 0,06 & 0 \\
\hline San Cayetano & 0,06 & 0 \\
\hline Zarate & 0,06 & 0 \\
\hline Chacabuco & 0,06 & 0,13 \\
\hline Chascomús & 0 & 0,07 \\
\hline $\begin{array}{l}\text { Coronel Suarez } \\
\text { González }\end{array}$ & 0 & 0,07 \\
\hline Chávez & 0 & 0,07 \\
\hline Laprida & 0 & 0,13 \\
\hline Luján & 0 & 0,07 \\
\hline Gral. Rodríguez & 0 & 0,07 \\
\hline Ramallo & 0 & 0,07 \\
\hline San Pedro & 0 & 0,07 \\
\hline TOTAL & 11,7 & $\mathbf{9 , 9 0}$ \\
\hline
\end{tabular}

Fuente: elaboración personal en base al Relevamiento Industrial Tandil. 2013. CIG-FCH-UNCPBA y Municipio de Tandil. 
La interacción urbana de Tandil con las localidades de la provincia de Buenos Aires presenta una directa relación con la jerarquía urbana preexistente. Así, como ya se ha señalado, el principal flujo de materias primas proviene de la RMBA al igual que el de insumos para la producción. Dentro de la RMBA, cabe destacar que estas mercaderías provienen principalmente de las localidades del sur de esa metrópolis (Avellaneda, Berazategui, Quilmes, entre otras) y muy escasamente de la ciudad capital. Se destacan la compra de las industrias textiles y de la fabricación de productos metálicos, maquinarias y equipos (rama 38) que adquiere en la RMBA chapa, aluminio, vidrio, acero, hiero, plástico, etc.

El volumen de compras de materias primas realizadas en la provincia de Buenos Aires por la industria tandilense es de 11,9\% y casi la mitad se realiza en la ciudad de Mar del Plata (6\%). Ésta actúa como una verdadera metrópolis regional para toda su región de influencia, en especial, por ofrecerse como una gran ciudad media que abastece a su región de todo tipo de productos, en especial, en lo referido a grandes compras mayoristas aunque también minoristas.

Las relaciones entre Tandil y Mar del Plata se vuelven cada vez más intensas ayudada además por la corta distancia entre ellas. Se destacan algunas ramas como son la 34, "fabricación de papel y productos de papel, imprentas y editoriales" cuyas principales materias primas son adquiridas en esa gran ciudad media sobresaliendo la compra de papel y tinta y el telgopor muy utilizado también en las ramas 33, 32, 37 y 38; las ramas 37 y 38 de la metalmecánica reciben flujos especialmente de bronce, hierro, chatarra, pinturas y aluminio.

La intensa y central interrelación interubana entre estas dos ciudades se observa además con la compra de insumos ya que la mitad de los flujos provinciales $(11,86 \%)$ provienen de Mar del Plata.

En cuanto a la circulación resultado de la necesidad de adquirir materias primas, a Mar del Plata le siguen las siguientes ciudades, según orden de importancia en cuanto al porcentaje que representan en el total del volumen de compras de materias primas en localidades de la provincia de Buenos Aires (exceptuando la RMBA): Balcarce (0,89\%), Bahía Blanca $(0,67 \%)$, Saladillo $(0,65 \%)$, Olavarría $(0,57 \%)$, Necochea $(0,4 \%)$, Chacabuco $(0,37 \%)$ y San Nicolás $(0,2 \%)$. (Ver tabla 02 y mapa 01$)$.

La ciudad de Balcarce se destaca por proveer harina para la industria alimenticia humana de Tandil y afrechiilo de trigo (que es un subproducto de la harina) utilizada para la 
producción de alimento balanceado. La especialización productiva de Balcarce, la cercanía a Tandil y, especialmente, la conectividad posible dada por la Ruta Nacional 226 son explicativas de esta importante relación de intercambio.

Bahía Blanca, es junto a Mar del Plata y La Plata, una de las tres ciudades más grandes e importantes de la red urbana provincial. Las relaciones económicas con esta ciudad, en cuanto a compras de materias primas, se explican por el Polo Petroquímico existente en esa ciudad, al adquirir gas carbónico para la elaboración de agua y soda y polietileno para la industria del plástico y derivados; también se destaca la compra de sémola.

El Partido de Saladillo (de un poco más de 30 mil habitantes según el Censo del INDEC 2010) y en particular su localidad cabecera cumple una función de proveedora de materias primas derivadas de la agricultura (cereales y oleaginosas especialmente) y producción de carne. En este sentido, las industrias productoras de chorizos y salamines declaran adquirir carne en Saladillo.

En estudios propios previos (LAN; LINARES; DI NUCCI e LOPEZ PONS, 2010) hemos escrito sobre la sub red urbana del centro de la provincia de Buenos Aires conformadas por Tandil-Olavarría-Azul las cuales se complementan por el papel urbano que cada una de ellas cumple. Esta interacción no es tan fuerte si miramos a Olavarría y Azul como proveedoras de materias primas para la industria de Tandil, aunque debemos destacar que Olavarría provee cemento a algunas industrias que fabrican hormigón armado y bloques de cemento, especialmente.

Respecto a las otras ciudades que muestran interrelación con Tandil a través de la compra en ellas de materias primas, podemos mencionar a Necochea, dónde se adquiere arena, arcilla y pelet de girasol (para la producción de alimento animal); Chacabuco, que provee especialmente harina y San Nicolás que vende a Tandil chapa para realizar perfiles y piezas fundidas de hierro de la industria metalmecánica.

Por último, como puede observarse en el mapa 01, Tandil compra materias primas en menores volúmenes a una gran cantidad de localidades pequeñas y medianas (veinticuatro en total) con las cuales mantiene una interrelación más débil como mercado comprador y vendedor. 


\section{Mapa 01: Flujos de materias primas (procedencia según volumen declarado) por} localidades de la provincia de Buenos Aires y RMBA (\%) 2013.

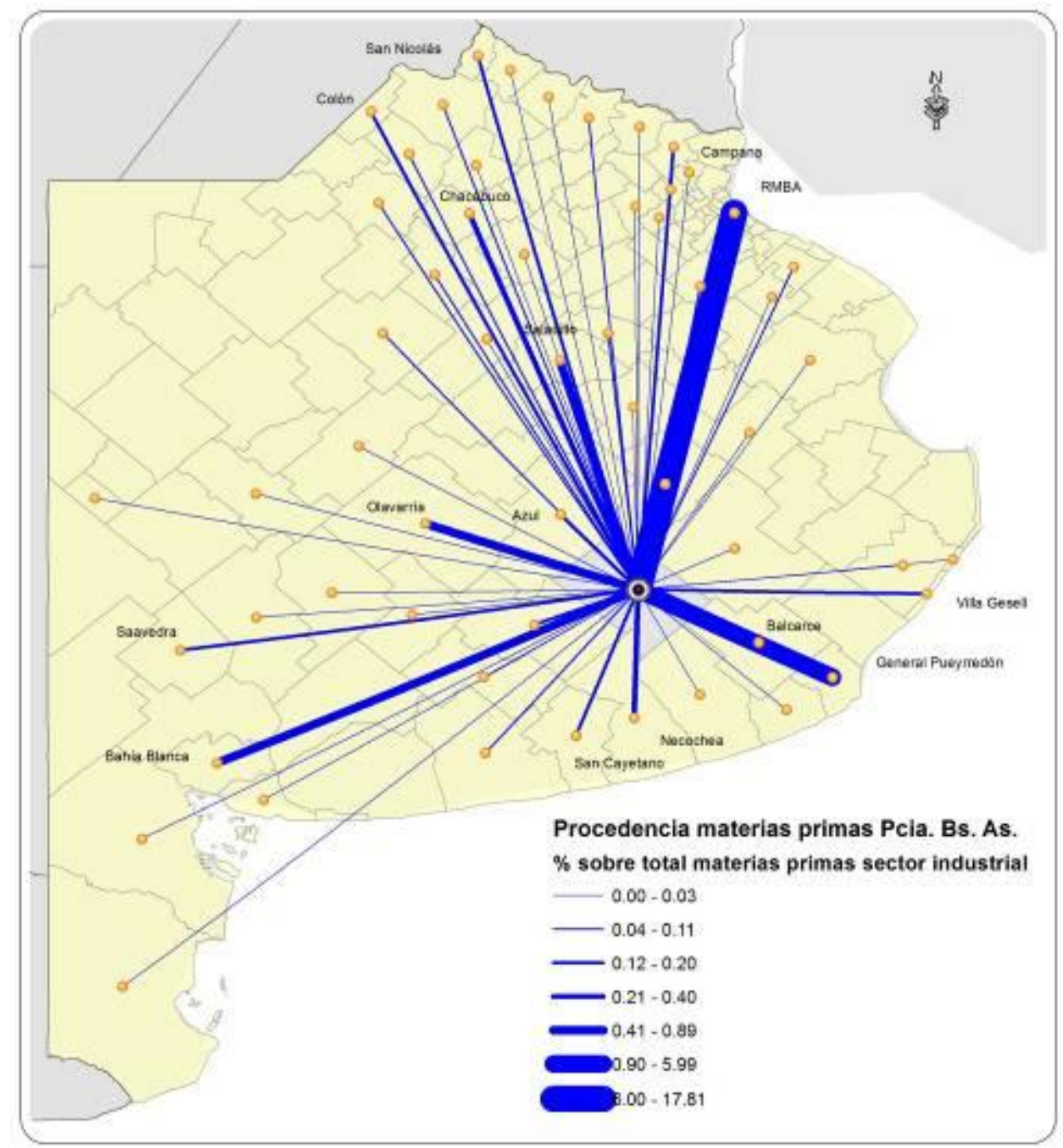

Fuente: elaboración personal en base al Relevamiento Industrial Tandil. 2013. CIG-FCH-UNCPBA y Municipio de Tandil

Los flujos de insumos necesarios para la industria de Tandil poseen un comportamiento bastante similar al de las materias primas en cuanto a la división interurbana del trabajo (BELTRÂO SPOSITO, 2009) ya que, en orden de importancia, son prácticamente las mismas ciudades las que abastecen a Tandil. Sin embargo cabe aclarar que estos flujos son menos permanentes o más fluctuantes que los de materias primas en cuanto a su procedencia.

Así, luego de Mar del Plata, los principales flujos de insumos provienen principalmente de Olavarría $(1,04 \%)$ a la cual le siguen Balcarce $(0,52 \%)$, Bahía Blanca $(0,39 \%)$, Benito Juárez $(0,26 \%)$, Junín $(0,26 \%)$, La Plata $(0,2)$ y Rauch $(0,2 \%)$. A estas 
localidades donde las compras son de mayor volumen, le siguen 17 localidades menores para las cuales también Tandil resulta ser parte de su mercado (Ver Tabla 02 y Mapa 02).

\section{Mapa 02: Procedencia de Insumos de la Industria de Tandil, por localidades de la} provincia de Buenos Aires (\%) 2013.

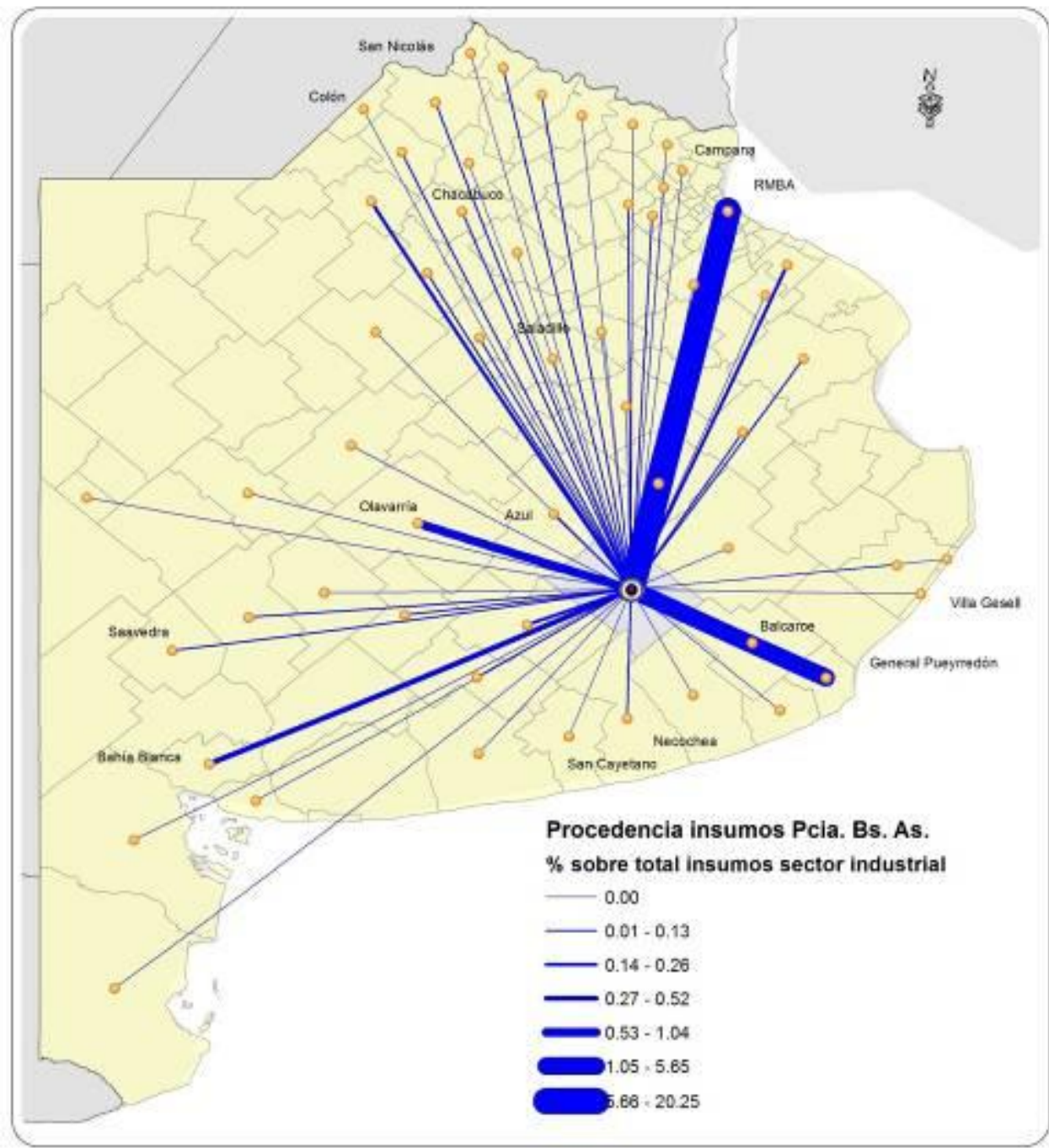

Fuente: elaboración personal en base al Relevamiento Industrial Tandil. 2013. CIG-FCH-UNCPBA y Municipio de Tandil.

Los flujos nacionales e internacionales de materias primas e insumos para la producción industrial

Volviendo a la tabla 01 podemos observar que la industria tandilense recibe flujos de varias localidades extraprovinciales, los que representan el 5,2\% de sus compras de materias primas y el 2,6\% de sus compras de insumos. En términos de Weber y su teoría de la localización industrial estaríamos hablando de materias primas localizadas, es decir, que solo 
se encuentran en un determinado punto y son esenciales para su elaboración. En este sentido, y según el informe realizado por Lan, D (2014), hay ramas industriales como la industria de la madera y productos de la madera, incluido muebles (rama 33) que son más dependientes de este tipo de mercancías hasta alcanzar el 20,6\% del total utilizado.

Una primera observación (tabla 03) nos permite mencionar que de ese 5,2\% correspondiente a las materias primas adquiridas en el resto de Argentina el 2\% se compran en la provincia de Santa Fe, continúan en orden de importancia las provincias de Entre Ríos y Córdoba (0,7\% cada una), Misiones $(0,6 \%)$ y Tucumán $(0,4 \%)$ y luego, en menor medida, las provincias de San Luis, Corrientes, Rio Negro, La Pampa, Mendoza, Chaco y Neuquén.

Ahora bien, pasando a una escala interurbana podemos observar en la tabla 03 y en el mapa 3 que se destacan las dos ciudades más grandes de Argentina tras Buenos Aires: Rosario y Córdoba. La importancia de la ciudad de Rosario (Santa Fe) está dada por la adquisición especialmente de lona por parte de los fabricantes locales de toldos; de acero, bronce y hierro por parte de la industria metalmecánica; de prolipropileno para la industria del plástico; de gas carbónico para la elaboración de agua y de arena de rio utilizada para la fundición.

Los flujos provenientes de Córdoba se explican principalmente por la compra de minerales para la fabricación de pintura, granito y piedras de pulir para elaboración de mesadas; chatarra, aluminio, hierro, electrodos y placas para la industria metalúrgica y tornerías; manteca para la elaboración de panificados y tinta para carteles.

Tucumán y Santa Fe son las grandes ciudades que continúan en orden de importancia, siendo interesante mencionar el caso de Tucumán ya que el flujo proveniente de ella es exclusivamente de azúcar, dadas las ventajas comparativas existentes y la localización de los principales ingenios azucareros en dicha provincia.

Cabe destacar algunas localidades como son San Pedro y El Dorado (provincia de Misiones) donde se adquieren maderas originarias para la industria local y, en menor medida, papel para la industria gráfica; localidades de la provincia de Entre Ríos, como Concordia y Federación, donde se compra también madera y Gualeguaychú de donde los principales flujos son de aserrín y pollos; este último producto es adquirido también en Villa Elisa. 
Tabla 03: Procedencia de Materias primas e insumos de la Industria de Tandil, por localidades del resto de Argentina (\%) 2013.

\begin{tabular}{|c|c|c|c|}
\hline Provincia & Localidad & $\begin{array}{c}\text { Materias } \\
\text { primas }\end{array}$ & Insumo \\
\hline \multirow[t]{7}{*}{ Santa Fe } & Bustinza & 0,1 & - \\
\hline & Las Parejas & 0,1 & - \\
\hline & Maciel & 0,1 & - \\
\hline & Rafaela & 0,2 & 0,3 \\
\hline & Rosario & 1,2 & 0,8 \\
\hline & Santa Fe & 0,4 & 0,2 \\
\hline & Venado Tuerto & 0,1 & - \\
\hline \multicolumn{2}{|l|}{ Total Prov. } & $\mathbf{2 , 0}$ & 1,2 \\
\hline \multirow[t]{4}{*}{ Entre Rios } & Concordia & 0,3 & - \\
\hline & Federación & 0,1 & - \\
\hline & Gualeguaychu & 0,2 & 0,1 \\
\hline & Villa Elisa & 0,1 & - \\
\hline \multicolumn{2}{|l|}{ Total Prov. } & 0,7 & 0,1 \\
\hline \multirow[t]{2}{*}{ Córdoba } & Córdoba & 0,7 & 0,5 \\
\hline & Marcos Juarez & - & 0,1 \\
\hline \multicolumn{2}{|l|}{ Total Prov. } & 0,7 & 0,5 \\
\hline Corrientes & Corrientes & 0,1 & - \\
\hline \multicolumn{2}{|l|}{ Total Prov. } & 0,1 & - \\
\hline \multirow[t]{2}{*}{ Misiones } & El Dorado & 0,2 & - \\
\hline & San Pedro & 0,4 & 0,1 \\
\hline \multicolumn{2}{|l|}{ Total Prov. } & 0,6 & 0,1 \\
\hline \multirow[t]{2}{*}{ Rio Negro } & General Roca & 0,1 & - \\
\hline & Ing. Jacobacd & 0,1 & 0,1 \\
\hline \multicolumn{2}{|l|}{ Total Prov. } & 0,1 & 0,1 \\
\hline \multirow[t]{2}{*}{ La Pampa } & Jacinto Arauz & 0,1 & - \\
\hline & Santa Rosa & 0,1 & 0,1 \\
\hline \multicolumn{2}{|l|}{ Total Prov. } & 0,1 & 0,1 \\
\hline Mendoza & Mendoza & 0,1 & - \\
\hline \multicolumn{2}{|l|}{ Total Prov. } & 0,1 & - \\
\hline Chaco & Resistencia & 0,1 & - \\
\hline \multicolumn{2}{|l|}{ Total Prov. } & 0,1 & - \\
\hline \multirow[t]{2}{*}{ San Luis } & Villa Mercedes & 0,1 & - \\
\hline & San Luis & 0,1 & - \\
\hline \multicolumn{2}{|l|}{ Total Prov. } & 0,2 & - \\
\hline \multirow[t]{3}{*}{ Neuquén } & Zapala & 0,1 & 0,1 \\
\hline & San Martín de los & - & 0,1 \\
\hline & Cinco Saltos & - & 0,1 \\
\hline Total Prov. & & 0,1 & 0,3 \\
\hline Tuaumán & Tucumán & 0,4 & 0,2 \\
\hline Total Prov. & & 0,4 & 0,2 \\
\hline San Juan & San Juan & - & 0,1 \\
\hline Total Prov. & & - & 0,1 \\
\hline Totales & & 5,2 & 2,6 \\
\hline
\end{tabular}

Fuente: elaboración personal en base al Relevamiento Industrial Tandil. 2013. CIG-FCH-UNCPBA y Municipio de Tandil. 
Hay algunas localidades con flujos menores pero que resultan de interés mencionar por la especialización que representan: la ciudad de Rafaela en la provincia de Santa Fe donde la más grande industria local de elaboración de helados adquiere leche; en la provincia de Rio Negro la localidad de Ingeniero Jacobacci, donde se encuentra un gran establecimiento minero que produce diatomea que son algas fósiles utilizadas en Tandil por empresas que fabrican productos refractarios; General Roca, donde se adquiere lúpulo para la fabricación cerveza y las localidades de La Pampa donde se adquiere cuero.

\section{Mapa 03: Procedencia de Materias primas de la Industria de Tandil, por localidades del} resto de Argentina (\%) 2013.



Fuente: elaboración personal en base al Relevamiento Industrial Tandil. 2013. CIG-FCH-UNCPBA y Municipio de Tandil. 
Con respecto a los insumos adquiridos en el resto de Argentina (tabla 03) se debe mencionar que este porcentaje, 2,6\%, es menor que el correspondiente a materias primas, aunque continúan destacándose las ciudades de Rosario y Córdoba seguidas por Santa Fe y Rafaela. Los productos adquiridos son similares a los explicados para el caso de materias primas pero en estos casos se utilizan como insumos.

Por último, respecto a los flujos de materias primas e insumos cabe hacer una mención a los flujos internacionales, es decir las importaciones, que si bien representan un pequeño porcentaje, $2 \%$ para materias primas y $2,4 \%$ para insumos (Tabla 04 ), hay ramas industriales o empresas locales para las cuales es central la adquisición de estos productos.

Así, si observamos los datos de dicha tabla encontramos cuales son los países con los cuales Tandil está conectada, en este caso por los flujos de materias primas e insumos. En lo respectivo a materias primas el primer lugar lo ocupa Alemania donde se adquieren maderas para fabricar pisos flotantes; sales para la elaboración de reactivos, perfilaría, PVC, aluminio y herrajes para la elaboración de aberturas de PVC y metal y plástico para la elaboración de cosechadoras.

China ocupa el segundo lugar en los flujos de materias primas a partir de las ventas de tinta y polifran para la elaboración de carteles; acaricidas sintéticos y orgánicos para elaborar productos apícolas y poliéster para fabricar productos textiles.

Si bien Brasil tiene el tercer lugar en el total de materias primas según procedencia extranjera, debe señalarse que hay ramas o empresas en particular donde su principal materia prima, en altos porcentajes, es comprada en ese país vecino. Existe de esta manera, una importante interacción espacial y hasta dependencia para algunas industrias locales siendo estos productos importados formadores de costos (elevados) para su producción. Algunos ejemplos son: la adquisición de acero por empresas locales representando el $60 \%$ del total de la materia prima necesaria para elaborar su producto que son moldes y la compra de chapas de acero inoxidable para elaborar quemadores para gas que representa el $70 \%$ de la materia prima necesaria para su fabricación. También, aunque en menor volumen, es destacable la importación de madera y nylon desde Brasil.

Otras materias primas importadas son: yute de India; melaninas de Suiza; toner de Japón; chapas de acero inoxidable de Francia; cables, componentes cerámicos y componentes electrónicos de Estados Unidos; fumagilina de Canadá y maderas de Bolivia y Paraguay. 
Con respecto a la importación de insumos por parte de las industrias tandilenes (ver tabla 04) se destaca China existiendo empresas que prácticamente importan de este país todos los principales insumos necesarios para su producción. Así, podemos mencionar vinilo y tinta para la elaboración de carteles; sulfato de amonio, metabelsufito de sodio, ácido forunco y ácido oxálico para fabricar suelas para calzado y flumetrina, cumafaz y amitraz para productos de sanidad apícola. Interesantes es destacar la compra de maquinarias, herrajes y plásticos en Alemania; tintas en Holanda y Corea y granaya en Italia, entre otros insumos.

Tabla 4: Procedencia de Materias primas e insumos para la producción industrial de Tandil por países del resto del mundo. 2013

\begin{tabular}{l|cc} 
Paises & $\begin{array}{c}\text { Materias } \\
\text { primas }\end{array}$ & Insumo \\
\hline Alemania & 0,55 & 0,45 \\
China & 0,43 & 0,84 \\
Brasil & 0,37 & 0,13 \\
EE UU & 0,18 & 0,26 \\
Canadá & 0,06 & 0,06 \\
Francia & 0,06 & 0,06 \\
India & 0,06 & 0,13 \\
Japon & 0,06 & 0,06 \\
Paraguay & 0,06 & 0 \\
Suiza & 0,06 & 0 \\
Bolivia & 0,06 & 0 \\
Italia & 0 & 0,19 \\
Corea & 0 & 0,13 \\
Holanda & 0 & 0,06 \\
Uruguay & 0 & 0 \\
\hline Totales & 1,96 & 2,4 \\
\hline
\end{tabular}

Fuente: elaboración personal en base al Relevamiento Industrial Tandil. 2013. CIG-FCH-UNCPBA y Municipio de Tandil.

\section{CONSIDERACIONES FINALES}

Presentamos en este trabajo una propuesta de estudio que ubica la fabricación o la producción propiamente dicha como parte del circuito espacial de producción de las empresas permitiendo llegar a estudiar la división territorial del trabajo de la industria local. 
Remarcamos la importancia de la circulación, del intercambio, en este caso de bienes materiales necesarios para la producción como son las materias primas y los insumos.

Quisimos ver las interacciones espaciales de Tandil en relación a su industria local en esa etapa previa a fabricar, es decir, en la adquisición de materias primas e insumos y como va formando parte de la red urbana preexistente al hacer uso y aprovecharla.

Se considera que conocer que materias primas utiliza Tandil, qué insumos necesita para su producción y dónde los adquiere es un conocimiento esencial del territorio que no solo deben tener las empresas sino todas instituciones que gestionan el territorio. Señalamos en especial los gobiernos en todas sus escalas, al momento de realizar y planear políticas industriales y comerciales, de decidir sobre el trazado y mejoras de rutas y accesos a nuestras ciudades, de decidir incentivos locales y extralocales a la producción, de incentivar o no ciertas iniciativas locales o inversiones externas, etc. Pero también instituciones que reúnen a los empresarios en sus diferentes estratos, como las cámaras empresariales o comerciales, ya que permitiría pensar y articular necesidades, dificultades, limitaciones o potencialidades.

En este sentido no puede perderse de vista que la industria local realiza un uso horizontal del territorio plasmado, en este caso, en la importancia del partido en la compra de materias primas e insumos. La fuerte horizontalidad mantenida por la industria de Tandil tiene que ser el foco sobre el cual se revean las políticas industriales muchas veces más preocupadas por incentivar las inversiones extralocales que en potenciar las inversiones locales existentes.

\section{REFERÊNCIAS}

ARROYO, Mónica. Redes e circulação no uso e controle do território. In: ARROYO, Mónica y DA CRUZ, Rita de Cássia Ariza (Org.). Territorio e circulação a dinâmica contradictoria da globalização. São Paulo: FAPESP/PPGH/CAPES/Ammablume Geografias, 2015. p. $37-49$.

ARROYO, Mónica y DA CRUZ, Rita de Cássia Ariza (Org.). Territorio e circulação a dinâmica contradictoria da globalização. São Paulo: FAPESP/PPGH/CAPES/Ammablume Geografias, 2015. p. $9-12$.

CORRÊA, Roberto Lobato. A rede urbana. São Paulo: Atica, 1989.

CORRÊA, Roberto Lobato. Interações espaciais. En: CASTRO, I. E de; GOMES, P. C da C.; CORREAA, R. L. (Orgs.). Explorações geográficas. Rio de Janeiro: Bertrand Brasil. 1997. p. 279-318. 
CORRÊA, Roberto Lobato. Estudos sobre a Rede Urbana. Rio de Janeiro: Bertrand Brasil. 2004.

LAN, Diana. Relevamiento Industrial de Tandil, 2013. Resultados generales.

CIG/FCH/UNCPBA y Municipalidad de Tandil. (Inédito). 2014.

LAN, Diana; LINARES, Santiago; DI NUCCI, Josefina; LOPEZ PONS, Magdalena. "La lógica de la organización espacial en la Ciudad de TANDIL" En: SPOSITO, M. Encarnação B., ELIAS, Denise, SOARES, Beatriz R. (Org.) Agentes econômicos e reestruturação urbana e regional: Uberlândia e Tandil. São Paulo: Editora Expressão Popular. 2010.

SANTOS, Milton. De la totalidad al lugar. Barcelona: Oikos - Tau. 1996.

SANTOS, Milton. La naturaleza del espacio. Técnica y tiempo. Razón y emoción. Barcelona: Ariel Geografía. 2000.

SILVEIRA, María Laura. Los territorios corporativos de la globalización. Geograficando, año 3, n. 3, p. 13- 26, 2007 [En línea]:

http://www.memoria.fahce.unlp.edu.ar/art_revistas/pr.3665/pr.3665.pdf

SILVEIRA, María Laura. Globalización y territorio usado: imperativos y solidaridades. Cuadernos del CENDES, año 25, n. 69, p. 1-19, septiembre-Diciembre 2008. [En línea]: http://www.scielo.org.ve/scielo.php?script=sci_arttext\&pid=S1012-25082008000300002

SPOSITO, Maria Encarnação Beltrão. Globalização, consumo e papéis intermediários de cidades médias no Brasil. In: BELLET SANFELIU, Carmen; SPOSITO, Maria Encarnação Beltrão (Org.). Las ciudades medias o intermedias en un mundo globalizado. Lleida: Edicions de la Universitat de Lleida, 2009, p. 41-69.

Recebido em Maio de 2016

Aprovado em Junho de 2016

Publicado em Junho de 2016

Sociedade e Território - Natal. Vol. 28, N. 1, p. 8-26. Jan./Jun. de 2016 